\title{
Avaliação dos perfis pressóricos, glicêmicos, lipêmicos, anêmicos e leucocitários de uma população atendida por projeto social na área metropolitana de Belém- PA
}

\author{
Evaluation of the pressure, glycemic, lipemic, anemic and leukocyte profiles of a \\ population assisted by a social project in the metropolitan area of Belém- PA
}

Evaluación de los perfiles de presión, glucémico, lipémico, anémico y leucocitos de una población asistida por un proyecto social en el área metropolitana de Belém- PA

Elem Cristina Rodrigues Chaves ${ }^{1 *}$, Kleber das Neves Trindade Júnior ${ }^{1}$, Oriana Karolina Corrêa Moraes $^{1}$, Alice Sachiko Araújo Yokoyama ${ }^{1}$, Felipe Gouvêa de Souza', Roberta Isabella Senna Ferreira $^{1}$, Ana Paula da Silva Ribeiro', Tinara Leila de Souza Aarão ${ }^{1}$, Michele Amaral da Silveira ${ }^{1}$, Maria Helena Rodrigues de Mendonça' ${ }^{1}$.

\section{RESUMO}

Objetivo: Avaliar os perfis pressóricos, glicêmicos, lipêmicos, anêmicos e leucocitários em uma população localizada na área metropolitana de Belém-PA. Métodos: Trata-se de um estudo transversal analítico, qualiquantitativo, composto por participantes de ambos os sexos e todas as faixas etárias. Resultados: Após análise, foi observado alterações na população à níveis pressóricos, com 57,1\% no sexo masculino e 42,9 no feminino. Esse mesmo perfil de alteração foi observado ao avaliar colesterol total $(44,4 \%)$, HDL $(54,4 \%)$ e LDL $(36,5 \%)$ do sexo masculino, entretanto, difere-se das alterações de triglicerídeos, com prevalência no feminino (11,5\%). Referente ao perfil anêmico e leucocitário, nota-se alterações em todos os paramétricos, com frequência de anemia branda em mulheres $(72,5 \%)$ e moderada em homens $(84,6 \%)$, além de macrocítica e hipocrômica na faixa $>12$ anos, e 2,3\% leucocitose em mulheres, 1,5\% de leucocitose e leucopenia em homens, tendo maior percentual a monocitopenia e eosinofilia. Conclusão: Tais achados expõem a vulnerabilidade das populações periféricas às doenças de base, principalmente a nível de condições nutricionais, habitacionais e educacionais. Com isso, torna-se relevante uma cooperação multiprofissional, via projetos e ações, para proporcionar às famílias o acesso à assistência básica e contribuir para qualidade de vida da população local.

Palavras-chave: Hipertensão, Diabetes mellitus, Dislipidemias, Anemias.

\begin{abstract}
Objective: To evaluate the pressure, glycemic, lipemic, anemic and leukocyte profiles in a population located in the metropolitan area of Belém-PA. Methods: This is an analytical, quali-quantitative cross-sectional study composed of participants of both sexes and all age groups. Results: After analysis, changes were observed in the population at blood pressure levels, with $57.1 \%$ in males and 42.9 in females. This same alteration profile was observed when evaluating total cholesterol (44.4\%), HDL (54.4\%) and LDL (36.5\%) males, however, differ from triglyceride alterations, with prevalence in females (11.5\%). Regarding the anemic and leukocyte profile, alterations were observed in all parametrics, with frequency of mild anemia in women $(72.5 \%)$ and moderate in men (84.6\%), in addition to macrocytic and hypochromic in the <a 12-year age group, and $2.3 \%$ leukocytosis
\end{abstract}

${ }^{1}$ Centro Universitário FIBRA. Belém - PA. *E-mail: elemcrc@gmail.com

SUBMETIDO EM: 6/2020

ACEITO EM: 6/2020

PUBLICADO EM: 9/2020

REAS / EJCH | Vol.12(9) | e4177 | DOI: https://doi.org/10.25248/reas.e4177.2020 Página 1 de 11 
in women, $1.5 \%$ leukocytosis and leukopenia in men, with a higher percentage of monocytopenia and eosinophilia. Conclusion: These findings expose the vulnerability of peripheral populations to underlying diseases, especially in terms of nutritional, housing and educational conditions. Thus, multiprofessional cooperation becomes relevant, through projects and actions, to provide families with access to basic care and contribute to the quality of life of the local population.

Keywords: Hypertension, Diabetes mellitus, Dyslipidemias, Anemias.

\section{RESUMEN}

Objetivo: Evaluar los perfiles de presión, glucémico, lipémico, anémico y leucocitos en una población ubicada en el área metropolitana de Belém-PA. Métodos: Se trata de un estudio transversal analítico y cuantitativo compuesto por participantes de ambos sexos y de todos los grupos de edad. Resultados: Después del análisis, se observaron cambios en la población a niveles de presión arterial, con $57,1 \%$ en los hombres y 42,9 en las mujeres. Este mismo perfil de alteración se observó al evaluar el colesterol total $(44,4 \%)$, HDL $(54,4 \%)$ y LDL $(36,5 \%)$ los machos, sin embargo, difieren de las alteraciones del triglicéridos, con prevalencia en las mujeres $(11,5 \%)$. En cuanto al perfil anémico y leucocitos, se observaron alteraciones en todas las paramétricas, con frecuencia de anemia leve en mujeres (72,5\%) y moderado en los hombres (84,6\%), además de macrocítico e hipocrómico en el <a grupo de edad de 12 años, y 2,3\% de leucocitosis en las mujeres, $1,5 \%$ de leucocitosis y leucopenia en los hombres, con un mayor porcentaje de monocitopenia y eosinofilia. Conclusión: Estos hallazgos exponen la vulnerabilidad de las poblaciones periféricas a las enfermedades subyacentes, especialmente en términos de condiciones nutricionales, de vivienda y educativas. Así, la cooperación multiprofesional cobra relevancia, a través de proyectos y acciones, para proporcionar a las familias acceso a la atención básica y contribuir a la calidad de vida de la población local.

Palabras-clave: Hipertensión, Diabetes mellitus, Dislipidemias, Anemias.

\section{INTRODUÇÃO}

Os acessos aos serviços de saúde, de forma universal e igualitária, constituem um dos direitos fundamentais de cidadania, sendo este garantido pelo Sistema Único de Saúde (SUS). No entanto, apesar de sua implementação e os avanços à ampliação dos serviços na rede básica de saúde, garantir o conjunto de princípios e diretrizes propostos pelo sistema constitui um desafio ao país, visto que, este sistema ainda se instala de forma seletiva, focalizada e excludente (OLIVEIRA RG, 2018).

Do ponto de vista sócio espacial, a sociedade está distribuída de maneira desigual quanto às características demográficas, desenvolvimento e riqueza, fato que reflete, frequentemente, nas condições de saúde em distintos grupos populacionais. Essa realidade repercute, no aumento da frequência de doenças e na redução da expectativa e qualidade de vida, sobretudo, de regiões e bairros periféricos com elevado nível de vulnerabilidade social, quer seja no acesso aos recursos e direitos básicos de qualquer cidadão ou no grau de risco a saúde (IPEA, 2018).

As doenças crônicas não transmissíveis (DCNT), relacionadas aos determinantes biológico, sociais e ambientais; a exemplo da diabetes tipo 2, obesidade, hipertensão arterial e dislipidemias, constituem-se como graves complicações, que influenciam no quadro de morbimortalidade e na qualidade de vida das pessoas acometidas, potencializando o risco de morte prematura e implicações econômicas às famílias, comunidades e sociedade de forma geral (MALTAI DC e SILVA MM, 2018; DOMINGUES JG, et al., 2019). Todavia, as DCNT atingem, com maior intensidade, os pertencentes a grupos vulneráveis de baixa renda e escolaridade, provocando incapacidades, limitações e redução da força produtiva, o qual contribui ainda mais para pobreza aos acometidos (DOMINGUES JG, et al., 2019).

Segundo Malta DC e Silva MM (2018), tais doenças impõem grandes custos socioeconômicos, estimados em US\$ 7 trilhões durante os anos de 2011 e 2025 em países de baixa e média renda. Nesse sentido, 
apresenta-se como um preocupante problema de saúde pública, que associada aos agravos e comorbidades de anemias, doenças reumáticas e parasitarias contribuem para o aumento do número de internações no SUS. O último inquérito realizado pelo Ministério da Saúde - Vigitel, aponta que esse aumento de custo associados a patologias preveníeis vêm onerando o sistema, desviando recursos que seriam aplicados em outras áreas da saúde (BRASIL, 2019).

A população periférica das grandes cidades é constantemente negligenciada referente à saúde pública, embora existam programas de promoção em saúde vinculada ao SUS, esses grupos populacionais encontram-se susceptíveis às doenças de base (IPEA, 2018). Diante disso, diversos fatores devem ser analisados com vista na prevenção e promoção à saúde em populações aos quais apresentam limitados recursos socioeconômicos e educacionais.

O monitoramento das DCNT e os fatores de riscos é essencial para reconhecer e entender sua distribuição e magnitude, com isso, para auxiliar na triagem dessas patologias, a realização de exames laboratoriais básicos consistem em poderosas armas da medicina para prevenir ou detectar doenças, sendo ferramentas essenciais na caracterização dos mais diversos perfis (GOLÇALVES J, et al., 2010; SANTOS LA, et al., 2015).

O perfil demográfico da população das áreas periféricas da cidade de Ananindeua é o reflexo da vulnerabilidade e disparidades social, o qual apresenta uma relação direta com o aparecimento de patologias como: diabetes, dislipidemias e anemias, visto isso, emergiu à necessidade de e implementar projeto a fim de aumentar o conhecimento do perfil dessa população e melhor propor novas ações em saúde no local.

Moldar cidades e moradores para promoção e prevenção a saúde é um desafio e requer esforço multidisciplinar que vão além das competências atuais dos setores de saúde. Nesse contexto, sendo as doenças não transmissíveis relacionadas principalmente as questões habitacionais, as intervenções em ambientes peridomiciliar e domiciliar são importantes frente ao combate e promoção a melhoria dos padrões de vida das populações.

Diante disso, o presente estudo teve por objetivo avaliar dos perfis glicêmicos, lipêmicos, anêmicos e leucocitários em uma população localizada na área metropolitana de Belém-PA.

\section{MÉTODOS}

Trata-se de um estudo transversal analítico, quantitativo e qualitativo, tendo como objeto de estudo participantes de ambos os sexos, de todas as faixas etárias que assinaram o Termo de Consentimento Livre e Esclarecido (TCLE) e o Termo de Assentimento (TA), e foram atendidos nas ações do Projeto "Ataque Total", cujo objetivo é realizar ações de Promoção Social e Cidadania, oferecendo serviços que atendam às necessidades das comunidades carentes em um município do estado do Pará. O Termo de Consentimento Livre e Esclarecido e Termo de Assentimento foi elaborado seguindo roteiro sugerido pelas Resoluções no 466/12 e 510/16 do Conselho Nacional de Saúde - CNS.

Por meio do Centro Universitário FIBRA, dentro do Projeto de Investigação Científica, foram realizadas três ações sociais do projeto "Ataque Total" no mês de agosto de 2019, setembro de 2019 e fevereiro de 2020 , respectivamente. No qual foram realizadas coletas de sangue, seguindo para realização dos exames no laboratório de análises clínicas.

Para análise dos determinantes de saúde biológicos: idade e sexo; e determinantes sociais e ambientais: nível de escolaridade e estilo de vida, foi utilizado um questionário sem solicitar identificação. Para aferição da pressão arterial (PA), foi utilizado o esfigmomanômetro, seguido da classificação de indivíduos hipertensos utilizando valores da PA obtidos no momento da aferição, adotando valores de referências da Sociedade Brasileira de Cardiologia (2016): $\leq 140$ e/ou $90 \mathrm{mmHg}$.

Quanto a investigação dos analíticos bioquímicos, foi utilizado sangue total, analisados pelo aparelho SX$3000 \mathrm{M}$ e os resultados foram comparados com valores de referências adotados pela Sociedade Brasileira de Cardiologia (2013): colesterol total (<200mg/dl), LDL ( $<100 \mathrm{mg} / \mathrm{dl}), \mathrm{HDL}(>60 \mathrm{mg} / \mathrm{dl})$ e triglicerídeos $(<150$ 
mg/dl), e pela Sociedade Brasileira De Diabetes (2019): Glicemia em jejum (<100 mg/dl). O hemograma foi realizado em equipamento Analisador Hematológico Automatizado com, no mínimo, 20 parâmetros.

Para o esfregaço, foram utilizados 10 microlitros de sangue homogeneizado, corado com o corante de Leishman e depois observado em microscópio óptico na objetiva de 100X, com valor de referência adotado pela Organização Mundial da Saúde (2011) e Hemograma manual de interpretação (FAILACE R, et al., 2015).

Como critério de inclusão, pacientes $>18$ anos que assinaram o TCLE e Crianças e Adolescentes que assinarem o TA com consentimento do responsável legal e doaram suas respectivas amostras e, critérios de exclusão, pacientes que não assinaram o TCLE, as amostras insuficientes (pouco volume), as amostras hemolisadas, no caso das bioquímicas e coaguladas, no caso do hemograma.

Para avaliação dos dados e formatação de tabelas, foi utilizado o Microsoft Excel versão 2016. Para fundamentação teórica, foi utilizado bases de dados online como Scientific Electronic Library Online (SciELO), Sistema Online de Busca e Análise de Literatura Médica (MEDLINE), Pan American Health Organization (PAHO), com os seguintes descritores: dislipidemias, anemia, hipertensão e Diabetes Mellitus.

O projeto recebeu aprovação do Comitê de Ética e Pesquisa (CAAE: 15457819.2.0000.8187; Parecer no: 3.439.261).

\section{RESULTADOS E DISCUSSÃO}

Após a realização de três ações sociais nos bairros do município de Ananindeua, foi obtido um $\mathrm{n}^{\circ}$ amostral de 297 participantes, desses, $67,3 \%$ são do sexo feminino e $32,7 \%$ masculino. Referente ao quantitativo de solicitações de exames laboratoriais, 78 são bioquímicos e 219 hematológicos.

Após análise dos dados, foi identificado 28 indivíduos hipertensos, com $57,1 \%$ no sexo masculino e $42,9 \%$ no feminino (Tabela 1/A). Quanto ao estado nutricional, considerando o Índice de Massa Corporal (IMC), foi possível observar alterações em ambos os sexos, todavia, com maior frequência no sexo masculino, com $100 \%$ de obesidade na faixa de $18-29$ anos e, somando-se as percentagens, $78,6 \%$ de sobrepeso e $59,5 \%$ de obesidade em maiores de 30 anos. Quanto ao sexo feminino, o maior percentual concentra-se em obesidade, com $71,4 \%$ na faixa de 30 a 49 anos e $75 \%$ em $>50$ anos (Tabela 1/B).

Referente ao nível de escolaridade, o ensino médio completo teve destaque entre os sexos, representando 57,1\% (30 a 39 anos) e 87,5\% (>50 anos) no feminino e 33,3\% (30 a 49 anos) e 57,1\% (>50 anos) no masculino, todavia, nesse destaca-se que $66,7 \%$ dos hipertensos na faixa de 18 a 29 anos possuem nível superior, demonstrando divergência com a literatura, pois expõe que o nível de ensino não exerce grande influência sob o quesito saúde.

Além disso, pode-se classificar uma elevada parcela da população como sedentários, visto que não praticam atividades físicas, sendo esse um fator primário de profilaxia à distintas doenças crônicas (Tabela 1/B). 
Tabela 1 - Perfil pressórico da população em estudo, onde A representa o quantitativo (n) e percentual de indivíduos considerados não hipertenso e hipertenso, segundo o sexo e B; distribuição dos hipertensos segundo determinantes biológicos, sociais e ambientais.

\begin{tabular}{cccccc}
\hline A Sexo & \multicolumn{2}{c}{ Não hipertenso } & \multicolumn{2}{c}{ Hipertenso* $^{*}$} & $\mathbf{N}$ \\
\cline { 2 - 5 } & $\mathbf{n}$ & $\%$ & 12 & 42,9 \\
\hline Feminino & 39 & 78 & 16 & 57,1 \\
Masculino & 11 & 22 &
\end{tabular}

\begin{tabular}{|c|c|c|c|c|c|c|}
\hline \multirow{3}{*}{ Variáveis } & \multicolumn{6}{|c|}{ Hipertenso (\%) $)^{*}$} \\
\hline & \multicolumn{3}{|c|}{ Feminino } & \multicolumn{3}{|c|}{ Masculino } \\
\hline & $18-29$ & $30-49$ & $>50$ & $18-29$ & $30-49$ & $>50$ \\
\hline \multicolumn{7}{|l|}{ Estado nutricional } \\
\hline Normal & 0 & 14,3 & 12,5 & 0 & 33,3 & 28,6 \\
\hline Sobrepeso & 0 & 14,3 & 12,5 & 0 & 50 & 28,6 \\
\hline Obesidade & 0 & 71,4 & 75 & 100 & 16,7 & 42,8 \\
\hline \multicolumn{7}{|l|}{ Escolaridade } \\
\hline Fundamental incompleto & 0 & 28,6 & 0 & 0 & $\begin{array}{ll}16,7 \\
\end{array}$ & $\overline{14,3}$ \\
\hline Fundamental completo & 0 & 14,3 & 12,5 & 33,3 & 16,7 & 14,3 \\
\hline Médio incompleto & 0 & 0 & 0 & 0 & 33,3 & 14,3 \\
\hline Médio completo & 0 & 57,1 & 87,5 & 0 & 33,3 & 57,1 \\
\hline Superior & 0 & 0 & 0 & 66,7 & 0 & 0 \\
\hline \multicolumn{7}{|l|}{ Atividade Física } \\
\hline Sim & 0 & 28,6 & 50 & 0 & $\begin{array}{ll}16,7 \\
\end{array}$ & 28,6 \\
\hline Não & 0 & 71,4 & 50 & 100 & 83,3 & 71,4 \\
\hline
\end{tabular}

Legenda: Período de coleta dos dados: agosto, setembro de 2019 e fevereiro de 2020; *Valores de referências adotados pela Sociedade Brasileira de Cardiologia (2016).

Fonte: Chaves ECR, et al., 2020.

De acordo com Botton A, et al. (2017) e Malta DC, et al. (2018), a prevalência de alterações pressórica no sexo masculino decorre da maior preocupação das mulheres com o bem-estar, aumentando sua procura pelos serviços de saúde, representando maior oportunidade de prevenção da hipertensão, enquanto que parte dos homens ainda se mantém refém do estigma da masculinidade, causando certa resistência à busca por auxílio médico e, por se sentirem invulneráveis, acabam expondo-se aos fatores de risco.

A hipertensão arterial sistêmica (HAS) é uma condição clínica multifatorial, todavia, é amplamente descrita na literatura sua associação com excesso de peso, sendo esse um fator de risco principalmente à indivíduos obesos e, em conjunto, a inatividade física que se destaca na população em estudo (WHO, 2012; RIBEIRO BB, et al., 2017; MALTA DC, et al., 2017).

De acordo com Ministério da Saúde (2019), cerca de 34.273 mil óbitos estão relacionados à diabetes, doenças cardiovasculares e câncer e, entre os fatores de risco, destaca-se o sedentarismo. Dados da Pesquisa de Vigilância de Fatores de Risco e Proteção para Doenças Crônicas por Inquérito Telefônico (Vigitel) expõem que apenas $37 \%$ dos brasileiros praticam atividade física, com maior frequência as crianças (BRASIL, 2019). Um fator encontrado nesse estudo, refere-se ao aumento no desenvolvimento de hipertensão e obesidade em adultos, sobretudo, idosos.

De forma geral, decorrem das alterações fisiológicas do envelhecimento, como maior resistência vascular, rigidez dos vasos sanguíneos, redução do metabolismo basal, diminuição da estatura e aumento da gordura corpórea, além de ser influenciado, como demonstra o Vigitel, com a menor prática de atividades físicas nesse público, corroborando maior exposição às doenças de base (RIBEIRO BB, et al., 2017; FERREIRA APS, et al., 2019). 
A partir das análises de colesterol total (CT), frações (HDL- colesterol e LDL- colesterol) e triglicerídeo (TG), foram encontradas alterações em ambos os sexos. Referente ao triglicerídeos, $11,5 \%$ encontram-se no sexo feminino, com $66,7 \%$ em $>20$ anos, e $11,1 \%$ no masculino, com $50 \%$ na faixa de 10 a 19 e $>20$ anos. Analisando o CT, HDL e LDL, o maior percentual de alterações concentram-se no sexo masculino com $44,4 \%$, $54,4 \%$ e $36,4 \%$, respectivamente, com distribuição de $83,3 \%$ a $100 \%$ em indivíduos $>20$ anos.

Quanto ao sexo feminino, o perfil etário se iguala ao masculino no tocante a CT e LDL $(78,9 \%$ e $72,7 \%)$, entretanto, $71,4 \%$ do HDL alterado está na faixa de 10 a 19 anos (Tabela 2/A). Analisando o perfil glicêmico da população, pode-se observar $20 \%$ das alterações em indivíduos de 30 a 49 anos, sendo todos masculino, e $50 \%$ em $>50$ anos, com $75 \%$ no sexo feminino e $25 \%$ masculino (Tabela $2 / B$ ).

Tabela 2 - Perfil lipídico e glicêmico da população em estudo, onde A demonstra a distribuição percentual dos valores normais e alterados de triglicerídeos, colesterol total e frações (HDL e LDL), segundo sexo e faixa etária e B; o quantitativo (n) e percentual de indivíduos com níveis de glicose normais e alterados segundo sexo (feminino- FEM e masculino- Mas) e faixa etária.

\begin{tabular}{|c|c|c|c|c|c|c|c|c|}
\hline \multirow{3}{*}{ A Variáveis* } & \multicolumn{8}{|c|}{ Sexo/faixa etária (\%) } \\
\hline & \multirow{2}{*}{ Total } & \multicolumn{3}{|c|}{ Feminino } & \multirow{2}{*}{ Total } & \multicolumn{3}{|c|}{ Masculino } \\
\hline & & $<9$ & $10-19$ & $>20$ & & $<9$ & $10-19$ & $>20$ \\
\hline \multicolumn{9}{|c|}{ Triglicerídeos $^{\star \star}$} \\
\hline Normal & 88,5 & 2,2 & 13 & 84,8 & 88,9 & 0 & 6,2 & 93,8 \\
\hline Alterado & 11,5 & 16,7 & 16,7 & 66,7 & 11,1 & 0 & 50 & 50 \\
\hline \multicolumn{9}{|c|}{ Colesterol total } \\
\hline Normal & 62,7 & 6,2 & 9,4 & 84,4 & 55,6 & 0 & 20 & 80 \\
\hline Alterado & 37,3 & 0 & 21,1 & 78,9 & 44,4 & 0 & 0 & 100 \\
\hline \multicolumn{9}{|l|}{$\mathrm{HDL}^{1}$} \\
\hline Normal & 83,7 & 2,8 & 13,9 & 83,3 & 45,5 & 0 & 0 & 100 \\
\hline Alterado & 16,3 & 0 & 71,4 & 28,6 & 54,5 & 0 & 16,7 & 83,3 \\
\hline \multicolumn{9}{|l|}{ LDL $^{2}$} \\
\hline Normal & 75 & 3 & 12,2 & 84,8 & 63,6 & 0 & 14,3 & 85,7 \\
\hline Alterado & 25 & 0 & 27,3 & 72,7 & 36,4 & 0 & 0 & 100 \\
\hline \multirow{2}{*}{ B Faixa etária } & \multirow{2}{*}{ Total (n) } & \multicolumn{7}{|c|}{ Glicose (\%) } \\
\hline & & & Normal & & Alterado & Fem/alterado & & alterado \\
\hline $0-17$ & 1 & & 100 & & 0 & 0 & & 0 \\
\hline $18-29$ & 4 & & 100 & & 0 & 0 & & 0 \\
\hline $30-49$ & 5 & & 80 & & 20 & 0 & & 100 \\
\hline$>50$ & 8 & & 50 & & 50 & 75 & & 25 \\
\hline
\end{tabular}

Legenda: Período de coleta dos dados: agosto e setembro de 2019 e fevereiro de 2020; *Valores de referências adotados pela Sociedade Brasileira de Cardiologia (2013); ${ }^{* *}$ Foi considerado o critério de sem jejum; ${ }^{1}$ High Density Lipoprotein; ${ }^{2}$ Low Density Lipoproteins. Fonte: Chaves ECR, et al., 2020.

Esses dados revelam um grau de dislipidemias e alterações a níveis de glicose, principalmente se tratando de comunidades carentes onde as condições socioeconômicas influenciam diretamente na resistência às mudanças de hábitos de vida, tornando-os vulneráveis a fatores de riscos agregados, essencialmente, a doenças cardiovasculares (DCV) (FUENMAYOR G, et al., 2013; MALTA CD, et al., 2019).

De acordo com a OMS, as DCV são consideradas a principal causa de morte a nível mundial, apresentam significativos gastos com assistência médica, atingindo principalmente indivíduos de baixa e média renda (OMS, 2017). Diversos estudos demonstram fatores de risco para jovens e adultos, sendo os principais hábitos alimentares inadequados, com consumo acentuado de gorduras totais, carboidrato e baixo ingestão de fibras alimentares, diabetes e o sedentarismo (FUENMAYOR G, et al., 2013; MASSA KHC, et al., 2019; SZWARCWALD CL, et al., 2019; SOUZA NA, et al., 2019).

Contrapondo-se a estudos realizados por Malta DC, et al. (2019) e Szwarcwald CL, et al. (2019) quanto a glicose, CT, HDL e LDL, embora o quantitativo de mulheres seja prevalente na população estudada, o sexo 
masculino apresenta a maior desregulação, entretanto, os estudos apresentam semelhanças quanto ao TG e a associação com aumento da idade. De acordo com a literatura, fatores como a menopausa e administração de hormônios, muito utilizados em >20 anos, podem contribuir para hipertrigliceridemia que, apesar de deter menos ênfase, há relatos da importância do triglicerídeos em pacientes com infarto do miocárdio pelo seu papel aterogênico (FRANCISCO AR, et al., 2016; KNOP LB, 2018; BRASIL, 2019).

As alterações de colesterol em $>20$ anos, concentrados no sexo masculino, podem relacionar-se a utilização de hormônios pela população feminina, principalmente através da alimentação (interferentes endógenos), a exemplo o estrógeno, o qual reduz os níveis de CT e LDL, causando aumento nos triglicerídeos e HDL (CLAPAUCH R, et al., 2002; FRANCISCO AR, et al., 2016). As maiores alterações encontram-se no público adulto, todavia, deve-se atentar-se aos jovens, uma vez que apresentaram um certo grau de alterações, principalmente a nível de HDL em mulheres de 10 a 19 anos e TG em homens nessa mesma faixa, dessa forma, destaca a manutenção da vida saudável como base para profilaxia da dislipidemia (OSAWA MS, et al., 2016; BRASIL, 2019).

De acordo com Silva HE, et al. (2018), parâmetro hematológico tem por objetivo avaliar os elementos celulares presentes no sangue, de maneira quali-quantitativa. Com isso, ao analisar os hemogramas da população em estudo, foi possível observar um certo grau alterações em todos os parâmetros hematológico, com variação de $1,4 \%$ a 79,9\% (Tabela 3).

Tabela 3 - Demonstra o quantitativo ( $n$ ) e a percentagem de indivíduos que apresentaram normalidades e alterações, de acordo com parâmetros hematológicos.

\begin{tabular}{|c|c|c|c|c|}
\hline \multirow{2}{*}{ Parâmetros hematológico* } & \multicolumn{2}{|c|}{ Normal } & \multicolumn{2}{|c|}{ Alterado } \\
\hline & $\mathbf{n}$ & $\%$ & $\mathbf{n}$ & $\%$ \\
\hline \multicolumn{5}{|l|}{ Eritrograma } \\
\hline Hemácia (/mm3) & 118 & 53,9 & 101 & 46,1 \\
\hline Hemoglobina $(g / d L)$ & 135 & 61,6 & 85 & 38,4 \\
\hline Hematócrito (\%) & 118 & 53,9 & 101 & 46,1 \\
\hline $\operatorname{VCM}(\mathrm{fL})$ & 66 & 30,1 & 153 & 69,9 \\
\hline $\mathrm{HCM}(\mathrm{pg})$ & 161 & 73,5 & 58 & 26,5 \\
\hline $\mathrm{CHCM}(\mathrm{g} / \mathrm{dL})$ & 44 & 20,1 & 175 & 79,9 \\
\hline RDW (\%) & 201 & 91,8 & 18 & 8,2 \\
\hline \multicolumn{5}{|l|}{ Leucograma } \\
\hline Leucócitos (/mm3) & 206 & 95,8 & 9 & 4,2 \\
\hline Neutrófilos (\%) & 159 & 74 & 56 & 26 \\
\hline Linfócitos (\%) & 172 & 80 & 43 & 20 \\
\hline Monócitos (\%) & 60 & 27,9 & 155 & 72,1 \\
\hline Eosinófilos (\%) & 149 & 69,3 & 66 & 30,7 \\
\hline Basófilo (\%) & 212 & 98,6 & 3 & 1,4 \\
\hline Plaquetas (/mm3) & 199 & 92,6 & 16 & 7,4 \\
\hline
\end{tabular}

Legenda: Período de coleta dos dados: agosto e setembro de 2019 e fevereiro de 2020; *Valor de referência adotado pela Organização Mundial da Saúde (2011) e Hemograma manual de interpretação (FAILACE R, et al., 2015); VCM - Volume Corpuscular Médio; HCM- Hemoglobina Corpuscular Média; CHCM - Concentração de Hemoglobina Corpuscular Média; RDW- Amplitude de Distribuição dos Glóbulos Vermelhos.

Fonte: Chaves ECR, et al., 2020.

Filho JDS, et al. (2017) apresenta a anemia sendo uma síndrome comum na prática clínica, desenvolvida diante uma infecção crônica, doença inflamatória crônica e neoplasias. Segundo a OMS (2017), as anemias são determinadas e classificadas de acordo com os níveis de hemoglobina, a qual sofre variação de acordo com sexo e faixa etária, devido a isso, considera-se esse parâmetro principal para epidemiologia das anemias. Estima-se que cerca de $42 \%$ das crianças com menos de 5 anos de idade e $40 \%$ das mulheres grávidas são afetados em todo o mundo (OMS, 2017).

Baseando-se na análise dos eritrogramas, especificamente, a nível de hemoglobina $(\mathrm{g} / \mathrm{dL})$, foi possível determinar indivíduos anêmicos e classificar a anemia de acordo com grau de severidade estabelecido pela OMS. De acordo com os dados, 64 apresentam-se anêmicos, sendo $62,5 \%$ e $20,3 \%$ no sexo feminino e 
masculino $>15$ anos, respectivamente, $11 \%$ em crianças $<11$ e $6,2 \%$ em adolescentes de 12 a 14 anos (Tabela 4/A). Referente ao grau de severidade, a maior percentagem é de anemia moderada concentrada em crianças de 5 a 11 anos (100\%), homens $>15$ anos $(84,6 \%)$ e $<4$ anos $(66,7 \%)$, contudo, as mulheres $>15$ anos prevalecem em anemia branda (72,5\%) e severa (2,5\%) (Tabela 4/A).

Avaliando o perfil morfológico eritrocitário, segundo os índices hematimétricos CHCM, VCM e RDW, foi observado prevalência na anemia macrocítica e hipocrômica $(46,9 \%)$ presente sobretudo em $>15$ anos e 12 a 14 anos, $51 \%$ e $50 \%$, respectivamente. Seguido de normocítica e hipocrômica $(28,1 \%)$ em $75 \%$ das crianças de 5 a 11 anos e $66,7 \%$ em $<4$ anos com presença de anisocitose e,15,6\% microcítica e hipocrômica em $<4$ anos $(33,3 \%)$ e $>15$ anos (17\%) com 33,3\% de anisocitose (Tabela 4/B).

Tabela 4 - Distribuição do quantitativo (n) e percentual dos indivíduos anêmicos por sexo e faixa etária, onde A demonstra a classificação da anemia de acordo com o grau de severidade à nível de hemoglobina (g/dL) e $\mathrm{B}$, os perfis morfológicos de acordo com índices hematimétricos (CHCM, VCM, RDW).

\begin{tabular}{|c|c|c|c|c|c|c|c|}
\hline \multirow{3}{*}{ A } & \multicolumn{7}{|c|}{ Anemia $^{1}$} \\
\hline & \multirow{2}{*}{ Total } & \multicolumn{2}{|c|}{ Branda } & \multicolumn{2}{|c|}{ Moderada } & \multicolumn{2}{|c|}{ Severa } \\
\hline & & $\mathbf{N}$ & $\%$ & $\mathbf{N}$ & $\%$ & $\mathbf{n}$ & $\%$ \\
\hline$<4$ & $3(4,8 \%)$ & 1 & 33,3 & 2 & 66,7 & 0 & 0 \\
\hline 5--11 & $4(6,2 \%)$ & 0 & 0 & 4 & 100 & 0 & 0 \\
\hline $12--14$ & $4(6,2 \%)$ & 2 & 50 & 2 & 50 & 0 & 0 \\
\hline Feminino $>15$ & $40(62,5 \%)$ & 29 & 72,5 & 10 & 25 & 1 & 2,5 \\
\hline Masculino $>15$ & $13(20,3 \%)$ & 2 & 15,4 & 11 & 84,6 & 0 & 0 \\
\hline \multirow[b]{2}{*}{ CHCM } & \multirow[b]{2}{*}{ VCM + RDW } & \multicolumn{4}{|c|}{ Anêmicos (\%) ${ }^{1}$} & \multicolumn{2}{|c|}{ Total } \\
\hline & & $<4$ & $5-11$ & $12-14$ & $>15$ & $\mathbf{n}$ & $\%$ \\
\hline \multirow{3}{*}{ Hipocromica } & Microcítica & 33,3 & 0 & 0 & $17^{\star *}$ & 10 & 15,6 \\
\hline & Normocítica & $66,7^{*}$ & 75 & 25 & 22,6 & 18 & 28,1 \\
\hline & Macrocítica & 0 & 25 & 50 & 51 & 30 & 46,9 \\
\hline \multirow{3}{*}{ Normocromica } & Microcítica & 0 & 0 & 0 & 0 & 0 & 0 \\
\hline & Normocítica & 0 & 0 & 25 & 1,9 & 2 & 3,1 \\
\hline & Macrocítica & 0 & 0 & 0 & 7,5 & 4 & 6,3 \\
\hline
\end{tabular}

Legenda: Período de coleta dos dados: agosto e setembro de 2019 e fevereiro de 2020; ${ }^{1}$ Valor de referência: Branda $<4$ anos $=10-19.9 \mathrm{~g} / \mathrm{dL}, 5-11$ anos $=11-11.4 \mathrm{~g} / \mathrm{dL},>12$ anos $=11-11.9 \mathrm{~g} / \mathrm{dL} ;$ moderada $<4$ anos $=7-$ $9.9 \mathrm{~g} / \mathrm{dL},>5$ anos $=8-10.9 \mathrm{~g} / \mathrm{dL}$; severa $<4$ anos $=<7 \mathrm{~g} / \mathrm{dL}$; $>5$ anos $=<8$ (OMS, 2011); Anisocitose em: 100\%*; 33,3\%**; VCM - Volume Corpuscular Médio; CHCM - Concentração de Hemoglobina Corpuscular Média; RDW - Amplitude de Distribuição dos Glóbulos Vermelhos.

Fonte: Chaves ECR, et al., 2020.

O perfil anêmico apresentado é amplamente descrito na literatura, destacando a maior prevalência de anemia em mulheres na idade reprodutiva ( 15 a 49 anos). De forma geral, as mulheres tendem a exibir uma reserva de ferro menor devido a distintos fatores como a menacme, gravidez, climatério e deficiência nutricional, todavia, não exclui possíveis condições patológicas (MARQUES F, et al., 2016; MACHADO IE, et al., 2019; PEREIRA SM, et al., 2019). Segundo Rodrigues LP, et al. (2010), durante a menacme há, em média, perda sanguínea de $25 \mathrm{~mL}$ a $30 \mathrm{~mL} / \mathrm{mês}$ ocasionando a diminuição de $12,5 \mathrm{mg}$ a $15 \mathrm{mg} / \mathrm{mês}$ ou $0,4 \mathrm{mg}$ a $0,5 \mathrm{mg} /$ dia de ferro, com isso, para que ocorra uma eritropoiese adequada, considerando a quantidade precisa de ferro $(5 \mathrm{mg} \mathrm{a} 10 \mathrm{mg} / \mathrm{dia})$, a necessidade desse mineral a essa população é elevada. Mesmo o maior percentual nesse público ser de anemia branca, os achados merecem destaque devido às consequências na qualidade de vida afetando principalmente o bem-estar e a capacidade de trabalho.

Contrapondo-se aos resultados de Machado IE, et al. (2019), mesmo o público masculino apresentando um menor percentual de anemia, estão mais frequentes em anemia moderada, ademais, outro ponto que merece destaque, refere-se ao elevado percentual de crianças anêmicas. Pode-se inferir que esse perfil se deve a uma maior necessidade de ferro devido à alta taxa de crescimento entre o sexo masculino não sendo, 
portanto, fornecido através da dieta (GOSWMAI S e DAS KK, 2015; SILVA PA, et al., 2018). Todavia, independente do grau de severidade, anemia na infância ocasiona implicações no desenvolvimento neuropsicomotor, capacidades físicas e de aprendizagem, além de gerar uma maior vulnerabilidade a infecções (SILVA PA, et al., 2018). Cerca de 90\% das anemias na infância, a nível mundial, referem-se à deficiência de ferro, sendo mais prevalência em sociedades de baixo nível socioeconômico (GOSWMAI S e DAS KK, 2015).

Os dados descrevem um perfil de hipocromia na população em estudo, a qual se estabelece devido a deficiência na síntese de hemoglobina originando células com diâmetro menor e coloração. Para biossíntese de hemoglobina, é essencial uma quantidade suficiente de ferro, sendo obtido através da dieta e reciclagem das hemácias senescentes clara (OMS, 2011). A presença da anemia microcítica e hipocrômica, presente principalmente em crianças, é clássico de anemias ferropênicas corroborando, portanto, a deficiência de ferro como fator primordial, todavia, não se exclui a possibilidade de anemia sideroblástica e talassemia que também apresentam essa morfologia, sendo necessário exames específicos. Ademais, para auxiliar nessa determinação, ressalta-se a presença de anisocitose, visto que a menor disposição do mineral causa um coorte eritrocitário, apresentando variação volumétrica e, com isso, RDW aumentado, sendo esse presente na população em estudo (MACEDO VF, et al., 2011; OMS, 2011).

Apesar desse importante perfil, a prevalência no estudo é macrocítica, normalmente associada a questões a nível de baixa absorção de cianocobalamina (Vitamina B12) e folato. Tais vitaminas são essenciais para formação de proteínas estruturais e hemoglobina sendo a alimentação uma rica fonte de obtenção, nesse sentido, reforça as deficiências nutricionais e seu impacto nessa população. Ademais, as alterações podem ser associadas ao alcoolismo, cirrose, hipotiroidismo e falência medular (MONTERO MD, et al., 2019).

Ao realizar a contagem global de leucócitos, foi possível observar $2,3 \%$ de leucocitose no sexo feminino com $60 \%$ em $>18$ anos e $40 \% 15$ a 17 anos; $1,5 \%$ de leucocitose e leucopenia no sexo masculino, o primeiro com $50 \%$ nas faixas de 5 a 11 anos e $>18$, o segundo com 100\% em $>18$ anos (Tabela 5). Na contagem diferencial, os sexos demonstraram similaridade quanto as alterações, tendo maior percentual a monocitopenia e eosinofilia. Na monocitopenia, as mulheres foram responsáveis por $48,7 \%$ e os homens $38 \%$, ambos em $>18$ anos, $73,3 \%$ e $44 \%$, respetivamente; na eosinofilia, o sexo masculino foi prevalente com $19,7 \%$ e o feminino com 18,5\% apresentando diferencia etária, com 34,6\% em homens de 5 a 11 anos e $57,5 \%$ em mulheres $>18$ anos. Ambos apresentaram plaquetopenia em>18 anos (Tabela 5).

Tabela 5 - Distribuição percentual dos tipos de alterações leucocitárias, de acordo com sexo e faixa etária, encontrados na população de estudo.

\begin{tabular}{|c|c|c|c|c|c|c|c|c|c|c|c|c|}
\hline \multirow{2}{*}{$\begin{array}{c}\text { Tipos de } \\
\text { alterações }\end{array}$} & \multirow{2}{*}{ Total } & \multicolumn{5}{|c|}{ Feminino (\%) } & \multirow{2}{*}{ Total } & \multicolumn{5}{|c|}{ Masculino (\%) } \\
\hline & & $<4$ & $5-11$ & $12--14$ & $15-17$ & $>18$ & & $<4$ & $5--11$ & $12--14$ & $15-17$ & $>18$ \\
\hline Leucocitose & 2,3 & 0 & 0 & 0 & 40 & 60 & 1,5 & 0 & 50 & 0 & 0 & 50 \\
\hline Leucopenia & 0 & 0 & 0 & 0 & 0 & 0 & 1,5 & 0 & 0 & 0 & 0 & 100 \\
\hline Neutrofilia & 3,2 & 14,3 & 14,3 & 0 & 0 & 71,4 & 3 & 75 & 25 & 0 & 0 & 0 \\
\hline Neutropenia & 12,5 & 0 & 3,7 & 7,4 & 11,1 & 77,8 & 13,7 & 5,6 & 16,7 & 22,2 & 11,1 & 44,4 \\
\hline Linfocitose & 6,5 & 0 & 0 & 7,1 & 7,1 & 85,8 & 9,8 & 7,7 & 15,4 & 15,4 & 0 & 61,5 \\
\hline Linfopenia & 3,2 & 14,3 & 42,9 & 0 & 0 & 42,9 & 6,8 & 44,4 & 44,4 & 0 & 0 & 11,2 \\
\hline Monocitose & 0 & 0 & 0 & 0 & 0 & 0 & 0 & 0 & 0 & 0 & 0 & 0 \\
\hline Monocitopenia & 48,7 & 3,8 & 9,6 & 5,7 & 7,6 & 73,3 & 38 & 12 & 28 & 10 & 6 & 44 \\
\hline Eosinofilia & 18,5 & 7,5 & 15 & 10 & 10 & 57,5 & 19,7 & 11,5 & 34,6 & 15,4 & 11,5 & 27 \\
\hline Basofilia & 0,5 & 0 & 0 & 0 & 0 & 100 & 1,5 & 0 & 0 & 0 & 0 & 100 \\
\hline Plaquetopenia & 4,6 & 10 & 10 & 0 & 0 & 80 & 4,5 & 1 & 1 & 1 & 0 & 3 \\
\hline
\end{tabular}

Legenda: Período de coleta dos dados: agosto e setembro de 2019 e fevereiro de 2020; *Valor de referência adotado pelo Hemograma manual de interpretação (FAILACE R, et al., 2015).

Fonte: Chaves ECR, et al., 2020.

Esse perfil leucocitário é descrito na literatura, principalmente envolvendo a leucocitose e leucopenia, porém, é raramente patognomônico pois pode ser influenciado por fatores distintos como resposta a infecções, estresse, utilização de medicamentos e traumatismo, assim como a monocitopenia que pode ocorrer devido ao estresse, uso de corticoides ou leucemia, no entanto, suas informações são úteis para 
estabelecer um diagnóstico diferencial, evolução e prognóstico de doenças (BATISTA AAP, et al., 2010; GOLÇALVES J, et al., 2010; SANTOS LA, et al., 2015).

Ademais, deve-se levar em conta as condições de vida existente de determinada população quanto as alterações da contagem diferencial dos leucócitos, uma vez que as péssimas condições de saneamento, higiene pessoal e/ou alimentação podem ser fatores determinantes em processo saúde-doença. A exemplo, a eosinofilia grandemente associada as infecções parasitarias que detém elevada prevalência no pais, particularmente em crianças, as quais interferem nos níveis nutricionais de ferro, como descrito no estudo (GOLÇALVES J, et al., 2010; SANTOS LA, et al., 2015). O leucograma é um importante instrumento de avaliação e investigação, sobretudo de infecções, sua implementação com frequência é de extrema importância na caracterização de perfil, principalmente, nas populações com precárias condições socioeconômicas, devido ao maior grau de exposição a fatores de risco.

\section{CONCLUSÃO}

A partir do cenário de elevado grau de alterações presente na população e em todas as variáveis analisadas, destaca-se más condições a níveis nutricionais, habitacionais e educacionais das populações. As políticas de gestão em saúde, em teoria, possuem boas estratégias para esses setores, no entanto, não conseguem suprir as demandas sociais, sobretudo, em localidades periféricas. Tais achados expõem a magnitude da vulnerabilidade social e sua contribuição as doenças de base, tornando essencial uma adequação dos serviços em saúde ao contexto atual das problemáticas sociais, além da cooperação multiprofissional, vinculada a projetos e ações, com vista a conhecer o perfil das populações e assim proporcionar as famílias o acesso à assistência básica, contribuindo para qualidade de vida da população local.

\section{REFERÊNCIAS}

1. BATISTA AAP, et al. Perfil Leucocitário De Pacientes Atendidos Em Um Laboratório Da Rede Pública De Saúde. Rev. Para. Med., 2010; 23(4).

2. BRASIL. Ministério da Saúde. Secretaria de Vigilância em Saúde. Departamento de Análise em Saúde e Vigilância de Doenças Não Transmissíveis - VIGITEL, 2019.

3. BRASIL. Ministério da Saúde. Protocolo Clínico e Diretrizes Terapêuticas Dislipidemia: prevenção de eventos cardiovasculares e pancreatite, 2019.

4. BOTTON A, et al. Diferenças de gênero no acesso aos serviços de saúde: problematizações necessárias. Mudanças: Psicologia da Saúde, 2017; 25(1):67-72.

5. CLAPAUCH R, et al. Fitoestrogênios: Posicionamento do Departamento de Endocrinologia Feminina da Sociedade Brasileira de Endocrinologia e Metabologia (SBEM) Arq Bras Endocrinol Metab, 2002; 46(6).

6. DOMINGUES JG, et al. Doenças crônicas não transmissíveis em profissionais de enfermagem de um hospital filantrópico no Sul do Brasil. Epidemiol. Serv. Saúde, Brasília, 2019; 28(2).

7. FAILACE R, et al. Hemograma: manual de interpretação. 6. ed. Porto Alegre: Artmed, 2015.

8. FERREIRA APS, et al. Prevalência e fatores associados da obesidade na população brasileira: estudo com dados aferidos da Pesquisa Nacional de Saúde. Rev Bras Epidemiol, 2019; 22 (1).

9. FILHO JDS, et al. Perfil hematológico e bioquímico de pacientes com doença de Chagas atendidos por um serviço de atenção farmacêutica no estado do Ceará J. Health Biol Sci. 2017; 5(2):130-136.

10. FRANCISCO AR, et al. Hipertrigliceridemia: há um papel para a aferese profilática? Relato de um caso J Bras Nefrol 2016; 38(3):366-369.

11. FUENMAYOR G, et al. Prevalência de dislipidemia em população infantil com cardiopatia congênita. Arq. Bras. Cardiol, 2013: 101(3):273-276.

12. GOLÇALVES J, et al. Perfil hematológico dos neonatos atendidos no Hospital Universitário da Universidade Federal de Santa Catarina. Rev. Bras. Hematol. Hemoter. 2010; 32(3):219-224.

13. GOSWMAI S, DAS KK. Determinantes socioeconômicos e demográficos da anemia infantilFatores socioeconômicos e demográficos determinantes da anemia infantil. Jornal de Pediatria, 2015; 91(5):471-477

14. INSTITUTO DE PESQUISA ECONÔMICA APLICADA-IPEA. Vulnerabilidade social no brasil: conceitos, métodos e primeiros resultados para municípios e regiões metropolitanas brasileiras. Brasília: Rio de Janeiro, 2018. 
15. KNOP LB. Efeito da menopausa e da hipertrigliceridemia na transferência de lípides e atividade da paraoxonase em mulheres diabéticas. Tese (Doutorado em Biotecnologia em Saúde e Medicina Investigativa) - FIOCRUZ. Salvador$\mathrm{BH}, 2018$.

16. MACEDO VF, et al. Prevalência de anemia em idosos de instituição de longa permanência em Brasília/DF. Geriatria \& Gerontologia. 2011; 5(4):214-9.

17. MACHADO IE, et al. Prevalência de anemia em adultos e idosos brasileiros Rev Bras Epidemiol, 2019; 22(2).

18. MALTA DC, et al. Prevalência e fatores associados com hipertensão arterial autorreferida em adultos brasileiros. Rev Saúde Pública. 2017; 51(1).

19. MALTAI DC, SILVA MM. A. S doenças e agravos não transmissíveis, o desafio contemporâneo na Saúde Pública. Ciênc. Saúde colet, 2018; 23(5).

20. MALTA DC, et al. Prevalência da hipertensão arterial segundo diferentes critérios diagnósticos, Pesquisa Nacional de Saúde. Rev Bras Epidemiol, 2018; 21(1).

21. MALTA DC, et al. Prevalência de colesterol total e frações alterados na população adulta brasileira: Pesquisa Nacional de Saúde, Rev Bras Epidemiol, 2019; 22 (2).

22. MASSA KHC, et al. Análise da prevalência de doenças cardiovasculares e fatores associados em idosos, 2000-2010 Ciência \& Saúde Coletiva, 2019; 24(1):105-114.

23. MARQUES F, et al. Contextualizando a Elevada Prevalência de Anemia na População Portuguesa: Perceção, Caracterização e Preditores: Um Sub-Estudo do EMPIRE Revista da sociedade portuguesa de medicina interna, 2016; 23(4).

24. MONTERO MD, et al. Anemia Megaloblástica: Revisão De Literatura. Revista Saúde em Foco, 2019; 11:934-963.

25. OLIVEIRA RG. Práticas de saúde em contextos de vulnerabilização e negligência de doenças, sujeitos e territórios: potencialidades e contradições na atenção à saúde de pessoas em situação de rua. Saúde Soc. São Paulo, 2018; 27(1):37-50.

26. ORGANIZAÇÃO MUNDIAL DA SAÚDE. Doenças cardiovasculares, 2017.

27. OSAWA MS, et al. Prevalência de fatores de risco de doença cardiovascular em trabalhadores de condomínios. Rev Bras Med Trab, 2016;14(2):108-114.

28. PEREIRA SM, et al. Estratégia de Saúde da Família e prevalência de anemia em mulheres de uma região urbana de alto Índice de Desenvolvimento Humano. J Hum Growth Dev, 2019; 29(3):410-415.

29. RIBEIRO BB, et al. Análise Hierarquizada da Hipertensão Arterial Sistêmica com a Variante Polimórfica do Gene da ECA e Outros Fatores de Risco em Idosos. International Journal of Cardiovascular Sciences. 2017; 30(1):52-60.

30. RODRIGUES LP, et al. Deficiência de ferro na mulher adulta. Rev. Bras. Hematol. Hemoter. 2010; 32(2):49-52.

31. SANTOS LA, et al. Perfil Leucocitário De Uma População Do Sul De Minas Gerais. Revista da Universidade Vale do Rio Verde, Três Corações, 2015; 13(1):506-513.

32. SILVA HE, et al. Hemogramas, 2018;1.

33. SILVA PA, et al. Associação entre a presença de anemia ferropriva com variáveis socioeconômicas e rendimento escolar. Medicina, 2018; 51(4):271-80.

34. SOCIEDADE BRASILEIRA DE CARDIOLOGIA. Diretriz Brasileira De Dislipidemias E Prevenção Da Aterosclerose, 2013; 101(4).

35. SOCIEDADE BRASILEIRA DE CARDIOLOGIA 7ª Diretriz Brasileira de Hipertensão Arterial, 2016;107(3):3.

36. SOCIEDADE BRASILEIRA DE DIABETES. Diretrizes da sociedade brasileira de diabetes 2019-2020. Clannad, 2020.

37. SZWARCWALD CL, et al. Valores de referência para exames laboratoriais de colesterol, hemoglobina glicosilada e creatinina da população adulta brasileira Rev Bras Epidemio, 2019; 22 (2).

38. WORLD HEALTH ORGANIZATION. World health statistics 2012. Geneva: WHO, 2012.

39. WORLD HEALTH ORGANIZATION. Nutritional anaemias: tools for eff ective prevention and control. Geneva: World Health Organization, 2017;83.

40. WORLD HEALTH ORGANIZATION. Haemoglobin concentrations for the diagnosis of anaemia and assessment of severity. Switzerland: Department of Nutrition for Health and Development (NHD), 2011. 